\title{
Stability of extended structures stabilized by light as governed by the competition of two length scales
}

\author{
Zhi Hong Hang, Jack Ng, and C. T. Chan* \\ Department of Physics, Hong Kong University of Science and Technology, Clear Water Bay, Hong Kong, China
}

(Received 16 November 2007; published 27 June 2008)

\begin{abstract}
Light-induced forces can bind dielectric spheres into extended structures through two mechanisms, each with its own length scale which can be adjusted by the configuration of the external light source. By manipulating the commensurability of the two length scales, these two mechanisms can cooperate to bind a large number of spheres. When the two length scales become incommensurate for some particular incident angle, the competition between the two mechanisms can lead to modulated structures and other complex phenomena such as reentrant stability.
\end{abstract}

DOI: 10.1103/PhysRevA.77.063838

PACS number(s): 42.50.Wk, 42.60.-v, 45.50.-j

\section{INTRODUCTION}

It is well established that light can be used as optical tweezers $[1,2]$ to grab and manipulate small particles. Light can also manipulate and bind a collection of microspheres into extended structures $[3,4]$, and the name "optical matter" has been proposed for these structures [5]. The evanescent fields from surface plasmons can also be used for optical binding in the literature $[6,7]$. Such structures can be regarded as a soft form of condensed matter with well defined form and structure, except that atoms are in this case replaced by microspheres, and the structures are bound by the exchange of photons rather than by chemical bonds. Mellor et al. have demonstrated experimentally that two competing mechanisms are at work [4]. The first is the optical gradient force, which is essentially a light-induced one-body force that traps a small dielectric particle at the maximum of the light intensity. This can be termed "optical trapping" (OT), and the interference pattern of the light will introduce a length scale $\lambda_{\mathrm{OT}}$. The second mechanism is the many-body optical force due to multiple scattering of light. This leads to "optical binding $(\mathrm{OB})$ " [8], which has its own inherent length scale $\lambda_{\mathrm{OB}}$ that depends on the relative phases of the induced dipoles on the particles. This multiple scattering mechanism can bind dielectric spheres into an ordered structure even if the external light source is homogenous in intensity [9]. Normally, $\lambda_{\mathrm{OT}}$ and $\lambda_{\mathrm{OB}}$ are not the same, and the competition between these two length scales can give rise to a host of interesting phenomena. The purpose of this paper is to examine the consequence when these two length scales operate either in synergy or in competition, leading to rather different results. The paper is organized as following. Sec. I is the introduction. In Sec. II, we introduce the two length scales induced by OT and OB and how and why the commensurability between the two length scales affects stability of optically bound structure. In Sec. III, we focus on incommensurate structures where interesting phenomena as reentrant stability and modulated structures are reported. The results are calculated using a fully fledged multiple scattering formalism and are interpreted using a simple model that cap-

\footnotetext{
*phchan@ust.hk
}

tures the essence of the physics. In Sec. IV, we conclude this work and in appendixes we give mathematics details omitted from main text.

\section{COMMENSURATE AND INCOMMENSURATE OPTICALLY BOUND SYSTEM}

This paper will focus on one-dimensional (1D) arrays. There have already been experimental [10-12] and theoretical [13-15] studies of 1D dielectric sphere chains. There have also been studies of 2D system of extended structures $[16,17]$. We will show that by manipulating the commensurability of the interference and scattering length scales $\left(\lambda_{\text {OT }}\right.$ and $\left.\lambda_{\mathrm{OB}}\right)$, it is possible to maximize the number of spheres that can be stabilized by light, and to obtain much longer stable chains than the usual transverse binding $[8,9]$ or longitudinal binding [10] configurations commonly considered in the literature. The somewhat counterintuitive results can be explained by simple models. Competition between the two length scales can also lead to the emergence of modulated structures.

The system considered is a chain of polystyrene spheres aligned along the $x$ axis (Fig. 1, inset), with the $i$ th sphere's position designated as $x_{i}$. Two pairs of equal-intensity counterpropagating electromagnetic waves of wavelength $\lambda$ and wave vectors $k$ lying in the same plane as the spheres illuminate the chain at an incident angle $\beta$. There is no net photon momentum. The incident field is then

$$
\begin{aligned}
\vec{E}_{\mathrm{inc}}= & \frac{1}{4} E_{0} \hat{y}\left(e^{i(k \cos \beta x+k \sin \beta z)}+e^{i(k \cos \beta x-k \sin \beta z)}\right. \\
& \left.+e^{i(-k \cos \beta x+k \sin \beta z)}+e^{i(-k \cos \beta x-k \sin \beta z)}\right) e^{i \omega t},
\end{aligned}
$$

where $k=\frac{2 \pi}{\lambda}$. The incident fields set an externally imposed length scale, as the beams interfere to form periodic fringes with a periodicity $\lambda_{\mathrm{OT}}=\frac{\lambda}{2 \cos \beta}$. However, the induced dipoles on adjacent spheres have a phase difference that depends on $\beta$ and in order for the dipole moment excited by the external field on one sphere to be in phase with the field scattered from the adjacent sphere, the adjacent spheres should be at a distance of $\frac{\lambda}{1 \pm \cos \beta}$. This sets a scattering length scale $\lambda_{\mathrm{OB}}$ $=\frac{\lambda}{1 \pm \cos \beta}$ for stable binding at arbitrary incident angles. The 


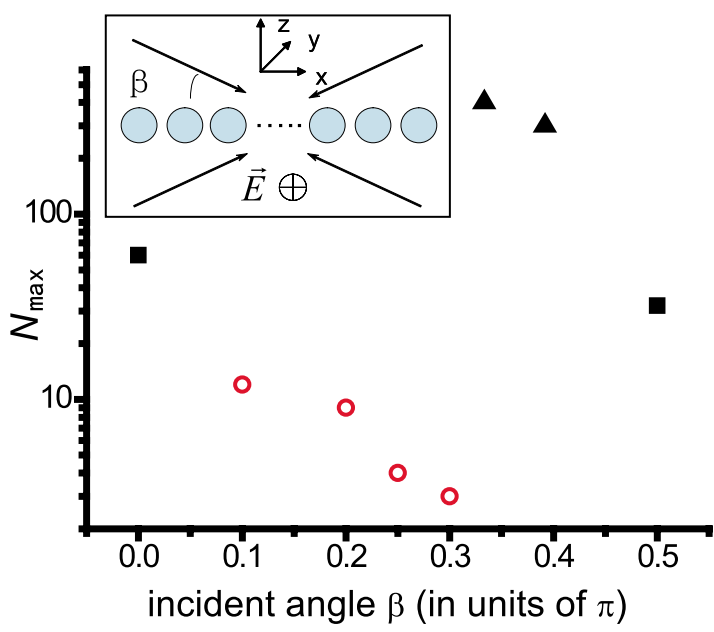

FIG. 1. (Color online) $N_{\max }$ is largest number of spheres that can be stabilized in a chain at different incident angles for $k a=1.1$. Incommensurate cases are shown with open circles while commensurate cases are shown with solid symbols. Note that there exist more stable configurations $\left(\beta=\cos ^{-1}\left(\frac{1}{3}\right)\right.$ and $\beta=\frac{\pi}{3}$, shown with triangles) other than longitudinal $(\beta=0)$ and transverse $(\beta=0.5 \pi)$ binding (shown with squares).

usual experimental configurations of longitudinal binding $\left(\beta=0, \lambda_{\mathrm{OT}}=\lambda_{\mathrm{OB}}=\frac{\lambda}{2}\right)$ and transverse binding $\left(\beta=0.5 \pi, \lambda_{\mathrm{OT}}\right.$ $=\infty, \lambda_{\mathrm{OB}}=\lambda$ ) correspond to configurations in which $\lambda_{\mathrm{OT}}$ and $\lambda_{\mathrm{OB}}$ are commensurate. However, at an arbitrary incident angle, $\lambda_{\mathrm{OT}}$ and $\lambda_{\mathrm{OB}}$ may not be commensurate, and the two length scales may compete. Such a system with two competing length scales can be considered as an optical analogy of the Frenkel-Kontorova (FK) model [18], although the onebody optical trapping force and the long-range interparticle scattering force have very complex forms and cannot be mapped directly to the FK model.

Rigorous multiple scattering (MS) and Maxwell stress tensor (MST) algorithm is used to analyze the 1D system of polystyrene $(\epsilon=2.53)$ spheres, whose radius $a$ is described in a dimensionless size parameter $k a=1.1$. For example, for a wavelength of $1064 \mathrm{~nm}$, the sphere diameter is $372 \mathrm{~nm}$. The binding force between two spheres of this size is about one $\mathrm{pN}$ at an incident light intensity of $0.01 \mathrm{~W} / \mu \mathrm{m}^{2}$. Spheres of radius larger than the wavelength have a different binding mechanism [11]. The MS-MST method solves the scattering fields for a given incident field configuration using the multiple scattering formalism and calculates the time-averaged forces using the Maxwell stress tensor. The details of the numerical method can be found in Ref. [9]. The results can be considered exact (up to numerical error) within a classical electromagnetic description. Molecular statics simulation is used to find zero-force positions for the collection of spheres, where the total field intensity is a local maximum. The force matrix $\overleftrightarrow{K}_{j k}=\frac{\partial\left(\vec{f}_{\text {light }}\right)_{j}}{\partial x_{k}}$ is evaluated numerically to determine stability near the zero-force configurations. For stable chains, the eigenvalues of the force matrix should be all negative or zero, as negative eigenvalues correspond to vibrations and zero eigenvalues correspond to neutral equilibrium (translation) $[9,19]$. If positive eigenvalues are found, the zero-force position is actually a saddle point, and the chain is unstable

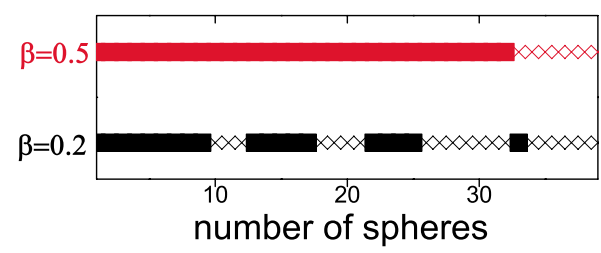

FIG. 2. (Color online) Stable (solid) and unstable (hatched) regions for the incommensurate configuration $\beta=0.2 \pi$ (black) and for the commensurate configuration $\beta=0.5 \pi$ (red).

in response to small perturbations. We constructed chains, found their zero-force positions and checked their stability with increasing number of spheres $N$ until $N_{\max }$ was reached, which was the largest number of spheres in the 1D chain that all chains with $N \leq N_{\max }$ were stable. For finite-sized spheres, each sphere cannot be exactly in phase due to finite size effect. Thus as $N$ increases, an unbinding mechanism due to multiple scattering will impose an upper limit $\left(N_{\max }\right)$ to the number of spheres that can be stabilized. As long as we are considering a linear response system, a more powerful laser cannot compensate for the unbinding mechanism. Figure 1 shows that $N_{\max }$ varies significantly with the incident angle $\beta$ (note that the vertical axis is in $\log$ scale). The variation can be explained by competition between $\lambda_{\mathrm{OT}}$ and $\lambda_{\mathrm{OB}}$. If the two binding mechanisms are to be commensurate, then $\frac{m \lambda}{2 \cos \beta}$ $=\frac{n \lambda}{1 \pm \cos \beta}$, which gives $\cos \beta=\frac{m}{2 n \mp m}$, where $m$ and $n$ are integers. The corresponding commensurate lattice constant is given by $a_{\text {com }}=(2 n \mp m) \frac{\lambda}{2}$. The usual experimental configurations of longitudinal binding corresponds to $m=1, n=1, \beta$ $=0$, while transverse binding corresponds to $(m=0, n=1, \beta$ $=0.5 \pi)$. Surprisingly, we managed to locate even more stable commensurate configurations. For example, when $(m=2, n$ $=1)$ and $(m=1, n=1)$, corresponding to $\cos \beta=\frac{1}{2}$ and $\cos \beta$ $=\frac{1}{3}$, more than 300 spheres can be stabilized into $1 \mathrm{D}$ chains whose lattice constants are $2 \lambda$ and $1.5 \lambda$, respectively. $N_{\max }$ is generally small for incommensurate configurations, such as for the incident angles of $0.1 \pi, 0.2 \pi, 0.25 \pi$, and $0.3 \pi$ (shown with circles). Thus, by adjusting incident angle to make the optical trapping and binding length scales commensurate, an extended chain with a large number of spheres can be stabilized.

\section{REENTRANT STABILITY AND MODULATED STRUCTURE}

Incommensurate configurations generate additional interesting effects, such as the emergence of modulated structures and the existence of reentrant stability. For commensurate configurations, chains with $N \leq N_{\max }$ are all stable, and we cannot find stable chains with $N>N_{\max }$. For the incommensurate configuration with $\beta=0.2 \pi, N_{\max }=9$, so that chains with one to nine spheres are all stable. Chains with $N=10$, 11 , or 12 are unstable, but other regimes of stability were found at $13 \leq N \leq 17,22 \leq N \leq 25$, and $N=33$. We cannot find stable chains with $N>33$. This is illustrated in Fig. 2.

In addition, the competition between OT and OB in incommensurate configurations gives rise to spatial modulations. In Figs. 3(a)-3(d), the separations between adjacent 


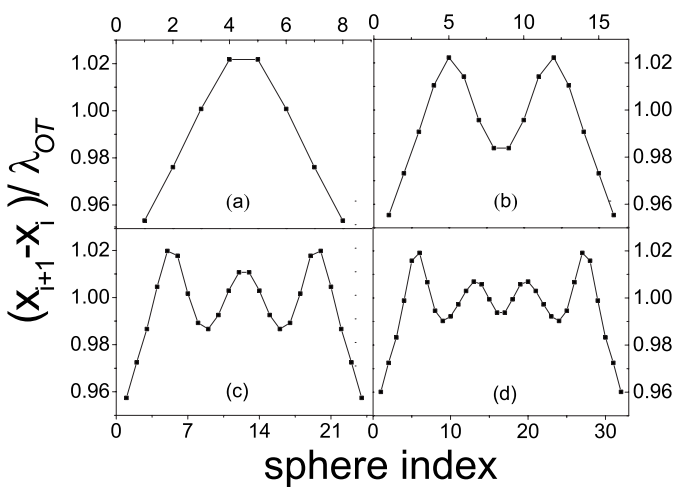

FIG. 3. Modulated structures for (a) $N=9$; (b) $N=17$; (c) $N$ $=25$; (d) $N=33$ chains at $\beta=0.2 \pi$ and $k a=1.1$. The horizontal axis is the sphere index $i$, and the vertical axis is the distance between adjacent spheres.

spheres are shown for the incident angle $\beta=0.2 \pi$ with $\mathrm{ka}$ $=1.1$ for four stable chain configurations with $N=9,17,25$, and 33 spheres. The average interparticle distance is about $\lambda_{\mathrm{OT}}$, plus a spatial modulation which repeats about every $8 \lambda_{\text {От. }}$. In contrast, commensurate configurations are always nearly periodic with a lattice constant $a_{\text {com }}$ as given above. The existence of a modulated structure is closely tied to the existence of multiple stable regimes. Apparently, chains of a length close to a multiple of the modulation $8 \lambda_{\text {OT }}$ are more stable, leading to the reentrant stability regimes for $N$ $>N_{\max }$. Figure 4 shows such modulated structures for various sphere sizes $(k a=0.6,0.9,1.1)$. The amplitude of the modulation clearly increases with sphere size, but the repeat distance remains the same and depends only on the incident angle.

The salient features of the numerical results can be explained with a dipole model. Consider a $1 \mathrm{D}$ chain of $N$ $=(2 n+1)$ spheres, each with polarizability $\alpha=\frac{4 \pi}{3} a^{3} \frac{\epsilon-1}{\epsilon+2}$. The position of the $i$ th sphere is $x_{i}$, where $i \in[-n, n]$. Symmetry demands that $x_{-i}=-x_{i}$. We truncate the scattering to the lowest order (the "Born approximation") to obtain closed form solutions. Within this approximation and for an incident standing wave, the potential energy can be defined as

$$
\begin{aligned}
U_{\text {total }}= & -\frac{\alpha}{4} \sum_{i}\left|E_{\text {inc }}\left(\vec{r}_{i}\right)\right|^{2}-\frac{\alpha^{2}}{2} \sum_{i} \sum_{j \neq i} \vec{E}_{\text {inc }}\left(\vec{r}_{i}\right) \\
& \times \operatorname{Re}\left[\overleftrightarrow{G}\left(\vec{r}_{i}-\vec{r}_{j}\right)\right] \cdot \vec{E}_{\mathrm{inc}}\left(\vec{r}_{j}\right),
\end{aligned}
$$

By inserting Eq. (1) into Eq. (2) and after some algebra (see Appendix A for details), we found that at equilibrium the distance between two particles are given by

$$
\begin{aligned}
x_{j+1}-x_{j} & =\alpha A \frac{\cos [2 \pi(1-\cos \beta) /(2 \cos \beta) j]}{j^{2} \pi^{2}}+\lambda_{\mathrm{OT}} \\
& =\frac{\alpha A}{j^{2} \pi^{2}} \cos \left(2 \pi j \frac{\lambda_{\mathrm{OT}}}{\lambda_{\mathrm{OB}}}\right)+\lambda_{\mathrm{OT}},
\end{aligned}
$$

where $A=\frac{k^{2}}{4 \pi \epsilon_{0}}$ is a constant and $\alpha \propto a^{3}$. We note that the dipole model is rather accurate for small spheres of size up to $k a \sim 1$ but should not be applied for larger spheres (see Ap-

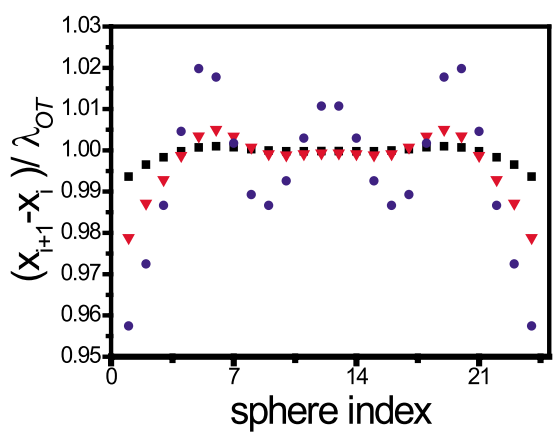

FIG. 4. (Color online) Modulated structures for $N=25$ chains and incident angle $\beta=0.2 \pi$, but with different sphere sizes: $k a$ $=0.6$ (squares), $k a=0.9$ (triangles), and $k a=1.1$ (circles).

pendix B). The radius of spheres should obviously not exceed $\lambda_{\mathrm{OT}} / 2(k a \sim 1.5)$, otherwise adjacent spheres will touch. Equation (3) shows that there is a modulation governed by $\frac{\lambda_{\mathrm{OB}}}{\lambda_{\mathrm{OT}}}$ on top of the periodic $\lambda_{\mathrm{OT}}$, and that its magnitude increases with the polarizability (i.e., with increasing sphere radius). When $\lambda_{\mathrm{OB}}$ and $\lambda_{\mathrm{OT}}$ are incommensurate, $\frac{2 \cos \beta}{1-\cos \beta}$ is irrational, and there is no way to avoid a modulated structure. The analytical results explain the results in Figs. 3 and 4 , and trace the emergence of a modulated structure to the competing length scales [20].

The dipole model predicts a specific dependence of the modulation on the incident angle, a prediction which was checked with full-fledged multiple scattering calculations for an $N=25$ chain at an incident angle $\beta=0.23 \pi$ and an $N$ $=22$ chain at $\beta=0.24 \pi$, both with $k a=1.1$. For $\beta=0.23 \pi$, the modulation periodicity was found to be about $6 \lambda_{\mathrm{OT}}$, while for $\beta=0.24 \pi$ the periodicity was about $5.25 \lambda_{\mathrm{OT}}$, as shown in Figs. 5(a) and $5(\mathrm{~b})$, respectively. The ratio of $\lambda_{\mathrm{OB}} / \lambda_{\mathrm{OT}}$ $=\frac{2 \cos \beta}{1-\cos \beta}$ is shown in Fig. 5(c) for comparison. $\lambda_{\mathrm{OB}}$ was found to be $6.00 \lambda_{\text {OT }}$ for $\beta=0.23 \pi$ and $5.38 \lambda_{\text {OT }}$ for $\beta=0.24 \pi$. Moreover, for $\beta=0.2 \pi, \lambda_{\mathrm{OB}}=8.27 \lambda_{\mathrm{OT}}$, which is close to the estimate of Fig. 3. The prediction of the simple model is thus qualitatively correct, and the modulated structure basically results from competition between OT and OB: OT provides the periodicity and $\mathrm{OB}$ induces a modulation.

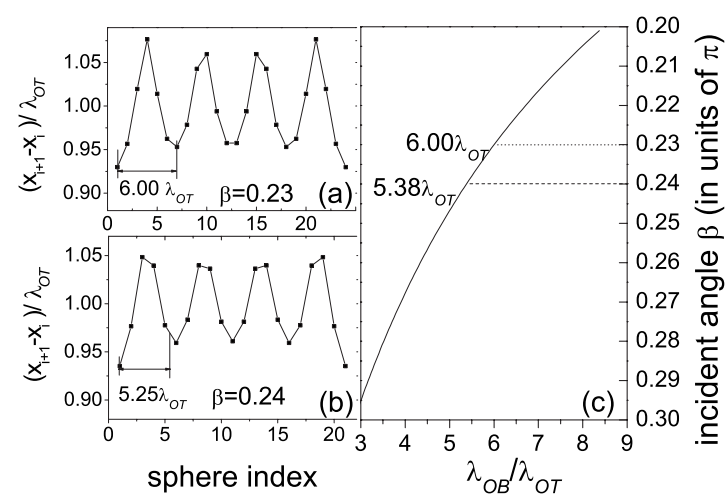

FIG. 5. Modulated structures for (a) $N=25$ chain at $\beta=0.23 \pi$; and (b) $N=22$ chain at $\beta=0.24 \pi$. The ratio of $\lambda_{\mathrm{OB}} / \lambda_{\mathrm{OT}}$ for different incident angles is shown in (c). 


\section{CONCLUSION}

In conclusion, light-induced forces have been shown capable of stabilizing dielectric spheres into 1D extended structures through the cooperative effect of a one-body optical trapping force and a scattering (optical binding) mechanism, each with its own length scale. If the incident angles can be tuned to obtain commensurate configurations, very long chains can be stabilized. If the incident light gives incommensurate configurations, the two length scales compete, leading to interesting effects such as modulated structures and reentrant stability.

\section{ACKNOWLEDGMENTS}

We thank Professor Z.F. Lin and Professor P. Sheng for many helpful discussions. We thank Professor Bain for providing unpublished results and helpful discussions. This work was supported by Central Allocation Grant No. HKUST3/06C. The computing resources involved were supported by Shun Hing Education and Charity Fund.

\section{APPENDIX A: DETAILED DERIVATION OF DIPOLE MODEL}

Consider a $1 \mathrm{D}$ chain of $N=(2 n+1)$ spheres, each with polarizability $\alpha=\frac{4 \pi}{3} a^{3} \frac{\epsilon-1}{\epsilon+2}$. The position of the $i$ th sphere is $x_{i}$, where $i \in[-n, n]$. Symmetry demands that $x_{-i}=-x_{i}$. We truncate the scattering to the lowest order (the "Born approximation") to obtain closed form solutions. Within this approximation and for an incident standing wave, the potential energy can be rigorously defined as [21]

$$
\begin{aligned}
U_{\text {total }}= & -\frac{\alpha}{4} \sum_{i}\left|E_{\mathrm{inc}}\left(\vec{r}_{i}\right)\right|^{2}-\frac{\alpha^{2}}{2} \sum_{i} \sum_{j \neq i} \vec{E}_{\mathrm{inc}}\left(\vec{r}_{i}\right) \\
& \times \operatorname{Re}\left[\stackrel{\leftrightarrow}{G}\left(\vec{r}_{i}-\vec{r}_{j}\right)\right] \cdot \vec{E}_{\mathrm{inc}}\left(\vec{r}_{j}\right) .
\end{aligned}
$$

It can be divided into two parts by its corresponding physical meaning:

$$
U^{\text {single }}=-\frac{\alpha}{4} \sum_{i}\left|E_{\text {inc }}\left(\vec{r}_{i}\right)\right|^{2}
$$

is the interaction between the external field and the induced dipole (OT) and

$$
U^{\text {interaction }}=-\frac{\alpha^{2}}{2} \sum_{i} \sum_{j \neq i} \vec{E}_{\mathrm{inc}}\left(\vec{r}_{i}\right) \operatorname{Re}\left[\stackrel{\leftrightarrow}{G}\left(\vec{r}_{i}-\vec{r}_{j}\right)\right] \cdot \vec{E}_{\mathrm{inc}}\left(\vec{r}_{j}\right)
$$

is the force due to scattering as communicated by the Green's tensor $\stackrel{\leftrightarrow}{G}\left(\vec{r}_{i}-\vec{r}_{j}\right)$ and represents OB. have

Inserting Eq. (1) into Eqs. (A2) and (A3), with $E_{0}=1$, we

$$
\begin{aligned}
U^{\text {single }} & =-\frac{\alpha}{4} \sum_{i=-n}^{n}\left[\cos \left(k \cos \beta x_{i}\right)\right]^{2} \\
& =-\frac{\alpha}{4} \sum_{i=-n}^{n}\left(\frac{1}{2}+\cos \left(2 k \cos \beta x_{i}\right)\right)
\end{aligned}
$$

and

$$
\begin{aligned}
U^{\text {interaction }}= & -\frac{\alpha^{2}}{2} \sum_{i} \sum_{j \neq i} \cos \left(k \cos \beta x_{j}\right) \cos \left(k \cos \beta x_{i}\right) \\
& \times \frac{1}{4 \pi \varepsilon_{0}\left|x_{i}-x_{j}\right|^{3}}\left\{\left(k^{2}\left|x_{i}-x_{j}\right|^{2}-1\right) \cos \left[k\left|x_{i}-x_{j}\right|\right]\right. \\
& \left.-k\left|x_{i}-x_{j}\right| \sin \left[k\left|x_{i}-x_{j}\right|\right]\right\} \\
= & -\alpha^{2} \sum_{i} \sum_{j<i} \cos \left(k \cos \beta x_{j}\right) \cos \left(k \cos \beta x_{i}\right) \\
& \times \frac{1}{4 \pi \varepsilon_{0}\left(x_{i}-x_{j}\right)^{3}}\left\{\left[k^{2}\left(x_{i}-x_{j}\right)^{2}-1\right] \cos \left[k\left(x_{i}-x_{j}\right)\right]\right. \\
& \left.-k\left(x_{i}-x_{j}\right) \sin \left[k\left(x_{i}-x_{j}\right)\right]\right\} .
\end{aligned}
$$

For simplicity, we keep only the lowest order dominant term of $1 / x_{i}-x_{j}$ in Eq. (A5) and it is further simplified to

$$
\begin{aligned}
U^{\text {interaction }}= & -\alpha^{2} \sum_{i} \sum_{j<i} \cos \left(k \cos \beta x_{j}\right) \cos \left(k \cos \beta x_{i}\right) \\
& \times \frac{k^{2} \cos \left[k\left(x_{i}-x_{j}\right)\right]}{4 \pi \epsilon_{0}\left(x_{i}-x_{j}\right)} .
\end{aligned}
$$

The equilibrium state of the chain requires the configuration of $\left\{x_{i}\right\}$ with lowest energy $U_{\text {total }}$ :

$$
\frac{\partial U^{\text {single }}}{\partial x_{i}}+\frac{\partial U^{\text {interaction }}}{\partial x_{i}}=0 \text { for all } x_{i} \text {. }
$$

For each $j \in[-n, n]$,

$$
\begin{aligned}
& \frac{\partial U^{\text {single }}}{\partial x_{j}}=\frac{\alpha}{2} k \cos \beta \sin \left(2 k \cos \beta x_{j}\right), \\
& \frac{\partial U^{\text {interaction }}}{\partial x_{j}}= \alpha^{2} \sum_{i=-n}^{j-1} A \cos \left(k \cos \beta x_{i}\right) \\
& \times\left\{k \cos \beta \sin \left(k \cos \beta x_{j}\right) \frac{\cos \left[k\left(x_{j}-x_{i}\right)\right]}{x_{j}-x_{i}}\right. \\
&+\cos \left(k \cos \beta x_{j}\right)\left(\frac{k \sin \left[k\left(x_{j}-x_{i}\right)\right]}{x_{j}-x_{i}}\right. \\
&+\left.\left.\frac{\cos \left[k\left(x_{j}-x_{i}\right)\right]}{\left(x_{j}-x_{i}\right)^{2}}\right)\right\}-\alpha^{2} \sum_{i=j+1}^{n} A \cos \left(k \cos \beta x_{i}\right) \\
& \times\left\{-k \cos \beta \sin \left(k \cos \beta x_{j}\right) \frac{\cos \left[k\left(x_{i}-x_{j}\right)\right]}{x_{i}-x_{j}}\right. \\
&+ \cos \left(k \cos \beta x_{j}\right)\left(\frac{k \sin \left[k\left(x_{i}-x_{j}\right)\right]}{x_{i}-x_{j}}\right. \\
&+\left.\left.\frac{\cos \left[k\left(x_{i}-x_{j}\right)\right]}{\left(x_{i}-x_{j}\right)^{2}}\right)\right\},
\end{aligned}
$$

where $A=k^{2} / 4 \pi \varepsilon_{0}$ is a constant.

Assuming that the OT is the stronger force, the solution should be of the form

$$
x_{j}=j \lambda_{\mathrm{OT}}+u_{j},
$$


where $u_{j}$ is a small perturbation. Inserting Eq. (A10) into Eqs. (A8) and (A9), we have

$$
\begin{aligned}
& \frac{\partial U^{\text {single }}}{\partial x_{j}}=\frac{\alpha}{2} k \cos \beta \sin \left(2 k \cos \beta u_{j}\right) \approx \alpha k^{2} \cos ^{2} \beta u_{j}, \\
& \frac{\partial U^{\text {interaction }}}{\partial x_{j}}=-A \alpha^{2} \sum_{i=-j+1}^{j-1} \frac{\cos \left(2 \pi \frac{1-\cos \beta}{2 \cos \beta}(j-i)\right)}{\left[\frac{\lambda}{2 \cos \beta}(j-i)\right]^{2}} .
\end{aligned}
$$

Inserting Eqs. (A11) and (A12) into Eq. (A7), we have

$$
\begin{gathered}
u_{j}=\alpha \frac{A}{\pi^{2}} \sum_{i=1}^{j-1} \frac{\cos [2 \pi(1-\cos \beta) /(2 \cos \beta) i]}{i^{2}}, \\
x_{j+1}-x_{j}=\alpha \frac{A}{\pi^{2}} \frac{\cos [2 \pi(1-\cos \beta) /(2 \cos \beta) j]}{j^{2}}+\lambda_{\mathrm{OT}} \\
=\frac{\alpha A}{j^{2} \pi^{2}} \cos \left(2 \pi j \frac{\lambda_{\mathrm{OT}}}{\lambda_{\mathrm{OB}}}\right)+\lambda_{\mathrm{OT}} .
\end{gathered}
$$

Equation (A13) is Eq. (3) in our paper.

\section{APPENDIX B: EFFECTIVENESS OF DIPOLE MODEL}

In this paper, we note that the numerical results are obtained using the highly precise multiple scattering method and we use the dipole model only for a easy understanding of the modulation and give qualitative understanding to the physical insight of the modulation. How accurate is the di-

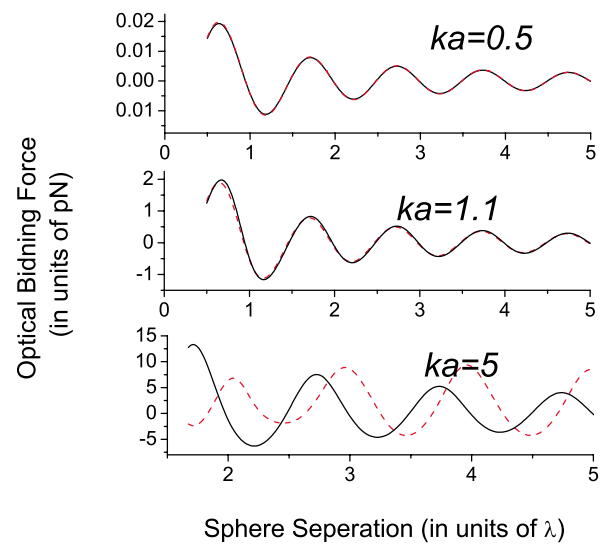

FIG. 6. (Color online) Optical binding force between two polystyrene spheres as the separation between two spheres changes. Force calculated by dipole model is in solid line and by MS-MST is shown as a dotted line.

pole model when optical forces are considered? For our full multiple scattering algorithm, the simulation will be precise for larger spheres $(k a>10)$ if large multipole numbers are taken into consideration. The dipole model is well known to work for sphere with $n k a \ll 1$, where $n$ is refractive index of sphere, and $k a$ is its size parameter. We are mainly discussing polystyrene $(n=1.59)$ sphere with $k a \sim 1$. Here, we compare the force between two polystyrene spheres under $\mathrm{OB}$ by $1064 \mathrm{~nm}$ laser at $0.01 \mathrm{~W} / \mu \mathrm{m}^{2}$ as the separation between two spheres changes (Fig. 6). A positive force is repulsive and negative force is attractive. For small spheres $(k a=0.5)$, dipole model gives the nearly exact results as MS-MST. For $k a=1.1$, the configuration we used mostly in our paper, it deviates about $3-5 \%$. For $k a=5$, the results are totally different. While the competition between OB and OT is always there for larger spheres; however, the result may deviate from dipole model predictions.
[1] A. Ashkin, Phys. Rev. Lett. 24, 156 (1970).

[2] D. Grier, Nature (London) 424, 810 (2003).

[3] M. MacDonald et al., Science 296, 1101 (2002).

[4] C. Mellor, T. Fennerty, and C. Bain, Opt. Express 14, 10079 (2006).

[5] M. M. Burns, J. M. Fournier, and J. A. Golovchenko, Science 249, 749 (1990).

[6] V. Garcés-Chávez, R. Quidant, P. J. Reece, G. Badenes, L. Torner, and K. Dholakia, Phys. Rev. B 73, 085417 (2006).

[7] M. Righini et al., Nat. Phys. 3, 477 (2007).

[8] M. M. Burns, J. M. Fournier, and J. A. Golovchenko, Phys. Rev. Lett. 63, 1233 (1989).

[9] J. Ng, Z. F. Lin, C. T. Chan, and P. Sheng, Phys. Rev. B 72, 085130 (2005).

[10] M. Guillon, O. Moine, and B. Stout, Phys. Rev. Lett. 96, 143902 (2006); M. Guillon, O. Moine, and B. Stout, ibid. 99, 079901(E) (2007).

[11] S. A. Tatarkova, A. E. Carruthers, and K. Dholakia, Phys. Rev. Lett. 89, 283901 (2002).

[12] W. Singer et al., J. Opt. Soc. Am. B 20, 1568 (2003).
[13] D. McGloin, A. E. Carruthers, K. Dholakia, and E. M. Wright, Phys. Rev. E 69, 021403 (2004).

[14] V. Karasek, K. Dholakia, and K. Zemanek, Appl. Phys. B: Lasers Opt. 84, 149 (2006).

[15] P. C. Chaumet and M. Nieto-Vesperinas, Phys. Rev. B 64, 035422 (2001).

[16] D. Maystre and P. Vincent, J. Opt. A, Pure Appl. Opt. 8, 1059 (2006).

[17] T. Grzegorczyk, B. Kemp, and J. A. Kong, J. Opt. Soc. Am. A 23, 2324 (2006).

[18] O. Braun and Y. Kivshar, The Frenkel-Kontorova Model: Concepts, Methods, and Applications (Springer, New York, 2004).

[19] J. Ng and C. T. Chan, Opt. Lett. 31, 2583 (2006).

[20] Here $\frac{2 \cos \beta}{1+\cos \beta}$ may also be a modulation periodicity when taking the plus sign of $\lambda_{\mathrm{OB}}$. But as $\cos \left(2 \pi \frac{1+\cos \beta}{2 \cos \beta} i\right)=\cos \left(2 \pi \frac{1-\cos \beta}{2 \cos \beta} i\right.$ $+2 \pi i)=\cos \left(2 \pi \frac{1-\cos \beta}{2 \cos \beta} i\right)$, these two correspond to the same kind of modulation.

[21] J. Ng, Ph.D. thesis, Hong Kong University of Science and Technology, Hong Kong, 2005. 